\title{
COMPARATIVE STUDY OF THE DYNAMICS OF CIRCULATING FLUIDIZED BED RISER AND DOWNER OF EQUAL DIAMETERS
}

\author{
S.V. Manyele \\ Chemical \& Process Engineering \\ College of Engineering and Technology \\ P. O. Box 35131, Dar Es Salaam \\ Email: smanyele@cpe.udsm.ac.tz
}

7

he microscopic flow dynamics of the 100-mm internal diameter riser/downer circulating fluidized bed (CFB) unit were monitored using a solids concentration fiber optic probe. The comparison was conducted by comparing radial profiles of the dynamic parameters derived from time-series analysis techniques (statistical and chaos analyses). The solids concentration signals used were recorded under similar operating conditions (gas velocity of $8.0 \mathrm{~m} / \mathrm{s}$ and solids flux of 100 $\mathrm{kg} / \mathrm{m}^{2} \mathrm{~s}$ ). Similar axial elevations were compared (entrance and fully developed sections of the two reactors). At each location, the signals were sampled at a frequency of 970 $\mathrm{Hz}$ for duration of 30 seconds. It was observed that the dynamical properties of the two reactors are similar in the core regions but different in the wall regions, mainly due to the turbulence caused by the up-flow of the gas-phase and down-flow of solids in the wall region of the riser. In general, the microscopic flow structure of the downer is more uniform than that of the riser.

Keywords: Multiphase reactor, riser, downer, solids concentration signal, time series analyses, chaos analysis, and circulating fluidized beds

\section{INTRODUCTION}

Fluidized bed reactors are the most applicable multiphase reactors in use in the industry for many years. Due to difficult design procedures, the understanding of the performance and the effect of different design features are mainly through extensive research efforts. This study is devoted to the dynamic behavior of the circulating fluidized beds (CFBs) monitored using fast sampling techniques.

The variations in the dynamic properties of a multiphase reactor can be caused not only by thermal or kinetic instabilities, or variations in an input such as feed flow rate (Hudson et al., 1990), but also by the difference in the flowdirection of the phases involved. Whether the phases flow against or in the same direction as the gravity can lead to many differences in the dynamic properties of the reactor. In this study, the differences and similarities between equaldiameter downer and riser of a CFB unit were investigated by studying the local solids concentration fluctuations in the two types of gas-solid reactors.

The downer reactor is a new type of fastfluidized beds where gas and solids flow concurrently downward assisted by gravity, different from risers where, the two phases flow upwards against gravity (Zhu et al., 1995). Research on the transient dynamics has been done separately for the two types of reactors using solids concentration signals from fiber optic probes. However, fewer studies have been conducted to compare the transient behavior between risers and downers, with limited data due to differences in configurations (height and diameters) and operating conditions (Tuzla et al., 1998; Chen et al., 1995). 
Most of the early research on particle movements was based on time-averaged particle velocities and solids concentration. From the transient records of solids concentration and pressure fluctuations, it is obvious that the particle movements are random. The dynamics of the CFBs show wide variations between different elevations due to entrance effect and flow development phenomenon. This study is focused on both dilute top and dense bottom sections of riser $(z / Z=0.054$ and 0.638$)$ and in the entrance and fully developed sections of downer $(z / Z=0.062$ and 0.655$)$. The dynamics of the bottom dense region of riser has received little attention probably because of its highly complex microflow structure. While other researchers consider the flow in the bottom region of the riser to be in turbulent regime (Bai et al., 1995; Bolton and Davidson, 1988) others regard it to be in bubbling fluidized bed regime (Svensson et al., 1996; Johnson et al., 1992).

In this study, the dynamical information contained within the signals is extracted using statistical analysis, cluster identification techniques (Manyele, et al., 2002) and chaos analysis (Schouten et al., 1994; van der Stappen, 1993a, 1993b; Daw and Halow, 1993; Grassberger and Proccaccia, 1983a, 1983b). Other parameters like spectral analysis (decay constant and amplitude of the spectrum in the lower frequency range) can also be used (Manyele et al., 2003). While statistical analysis does not reveal time dependency of the flow in the two different kinds of reactors, chaos analysis provides information on both time dependency and complexity at a microscopic level.

Calculating the correlation dimension, $D$, and the Kolmogorov entropy, $K_{\mathrm{MI}}$, of the multidimensional reconstructed attractor using a single signal, can reveal the differences between riser and downer based on complexity in phase space or the number of dynamical degrees of freedom and the rate of information loss or gain as the gas and solids flow. Cheng et al. (1995) compared the downer and riser using local chaotic behavior from solids concentration data also from a fiber optic probe as summarized in Table 1. Their results show remarkable differences between downer and riser in terms of

Table 1: Equipment and operating conditions used by different investigators for comparing flow dynamics between downer and riser

\begin{tabular}{|c|c|c|c|c|c|c|}
\hline \multirow[t]{2}{*}{ Parameter } & \multicolumn{2}{|l|}{ This work } & \multicolumn{2}{|c|}{ Tuzla et al. (1998) } & \multicolumn{2}{|c|}{ Cheng et al. (1995) } \\
\hline & Downer & Riser & Downer & Riser & Downer & Riser \\
\hline Diameter [mm] & 100 & 100 & 150 & 150 & 92 & 140 \\
\hline Height $[\mathrm{m}]$ & 9.5 & 15.7 & 9 & 10 & 4 & 10.4 \\
\hline$U_{g}[\mathrm{~m} / \mathrm{s}]$ & 8.1 & 8.0 & 6 & 5 & $1-3$ & $1-3$ \\
\hline$G_{s}\left[\mathrm{~kg} / \mathrm{m}^{2} \mathrm{~s}\right]$ & 101 & 100 & 89 & 89 & $20-50$ & $20-50$ \\
\hline$d_{p}[\mu \mathrm{m}]$ & 67 & 67 & 125 & 250 & 54 & 54 \\
\hline Solids type & $\mathrm{FCC}$ & $\mathrm{FCC}$ & GB & GB & $\mathrm{FCC}$ & $\mathrm{FCC}$ \\
\hline$\rho_{p}\left[\mathrm{~kg} / \mathrm{m}^{3}\right]$ & 1600 & 1600 & - & - & 1398 & 1398 \\
\hline \multirow{2}{*}{$z / Z$} & 0.054 & 0.062 & - & - & - & - \\
\hline & 0.655 & 0.638 & 0.464 & 0.5 & 0.913 & 0.298 \\
\hline $\begin{array}{l}\text { Measurements } \\
\text { of } \varepsilon_{\mathrm{s}}(\mathrm{t})\end{array}$ & $\begin{array}{l}\text { Reflective } \\
\text { optic probe }\end{array}$ & type fiber & \multicolumn{2}{|c|}{$\begin{array}{l}\text { Needle capacitance } \\
\text { probe }\end{array}$} & \multicolumn{2}{|l|}{$\begin{array}{l}\text { Optical } \\
\text { probe }\end{array}$} \\
\hline Sampling rate & \multicolumn{2}{|l|}{$970 \mathrm{~Hz}$} & \multicolumn{2}{|l|}{$5000 \mathrm{~Hz}$} & \multicolumn{2}{|l|}{$420 \mathrm{~Hz}$} \\
\hline
\end{tabular}


radial profiles of chaotic parameters $(D$ and $K_{\mathrm{ML}}$ ). The drawback of their results lies on the fact that they utilized very low solids flux, $\left(G_{\mathrm{s}}=\right.$ $\left.20-50 \mathrm{~kg} / \mathrm{m}^{2} \mathrm{~s}\right)$, which for a corresponding velocity range of $1-7 \mathrm{~m} / \mathrm{s}$, the conditions were excessively dilute. Thus more investigation is still needed especially for operating conditions employing higher $G_{\mathrm{s}}\left(100-200 \mathrm{~kg} / \mathrm{m}^{2} \mathrm{~s}\right)$ and high flux conditions $\left(G_{s}>200 \mathrm{~kg} / \mathrm{m}^{2} \mathrm{~s}\right)$.

The distribution of the solid-phase in the fastfluidized bed is strongly influenced by the tendency of solid particles to aggregate into dynamic clusters, which flow with very different velocities from that of the individual particles. The clusters change continuously in terms of size, density and velocity. As a result, variations occur in local solids concentration from the average values as the clusters move up or down. A number of researchers have investigated this property of solids flow (Manyele et al, 2002; Tuzla et al., 1998; Soong et al., 1993) as shown in Table 1. Despite of these efforts most of the investigations were based on risers and very limited work is reported for downers. Furthermore, no attempts have been made to compare the characteristics of clusters between downer and riser. Tuzla et al. (1998) employed a needle-type capacitance probe in detecting the dynamic variations of local solids concentration in both downer and riser. They employed statistical analysis and compared the cluster tendency between the two types of reactors. Because of differences in particle size of the glass beads used in riser and downer, and a wide gap between the operating conditions, it was difficult to make a clear conclusion. Moreover, their study utilized very low solids circulation rates $\left(G_{s}<60 \mathrm{~kg} / \mathrm{m}^{2} \mathrm{~s}\right)$.

This study is important as it address the comparison at similar operating conditions, similar diameters and using the same solid particles (spent FCC powder). As shown in Figure 1, the two units are connected together and can only be operated at the same time.

\section{EXPERIMENTAL SETUP}

Table 1 summarizes the design features and operating conditions used in this study. Similar operating conditions were employed in both riser and downer of the same internal diameter, with same type of solid particles recirculated between the two reactors (see Figure 1, Manyele et al, 2003). Measurements of solids concentration were performed using a fine optical fiber probe (Zhang et al., 1998). Spent FCC powder ( $d_{p}=67$ $\mu \mathrm{m}$ and $\rho_{p}=1600 \mathrm{~kg} / \mathrm{m}^{3}$ ) was circulated between the two units. Measurements of the solids circulation rate (or solids flux), $G_{s}$, were performed at a single point in the circulation loop (using a measuring vessel).

\section{THEORY AND DATA PROCESSING TECHNIQUES}

The important statistical parameters used in this study include the time-averaged values and standard deviation determined from the signal, $\varepsilon_{\mathrm{S}}(t)$, with $N=27,100$ data points sampled within 30 seconds. Based on the properties of the signals from solids concentration fiber optic probe, two parameters were further derived, i.e. the number of cycles, $N_{c}$, and the average cycle time (ACT), denoted as $T_{a c}$. The number of cycles was determined as half the number of times the signal crosses its mean value up and down. Given the number of cycles, the average cycle time, $T_{a c}$, is defined by van der Stappen et al. (1993b) as per equation

$T_{a c}=\frac{2}{N_{c}} \sum_{i=1}^{N_{c}}\left(\Delta T_{c i}\right)$

where $\Delta T_{c}$ is the time interval between crossings over the mean (in $\mathrm{sec}$ ), and $N_{c}$ is the total number of crossings (upward and downward). 


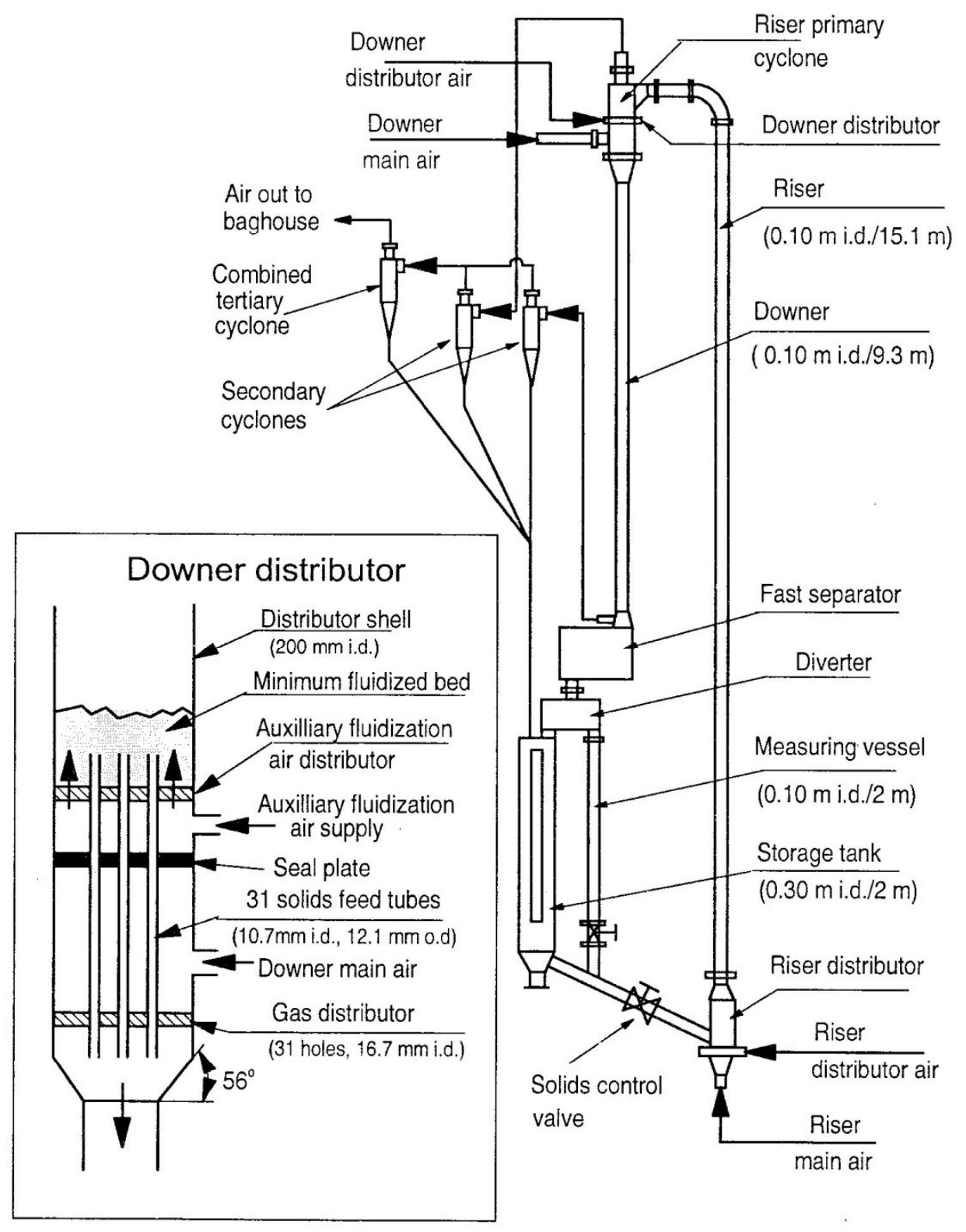

Figure 1: Experimental setup for gas-solids circulating fluidized bed showing the downer reactor and its accessories (Manyele et al., 2003).

The ACT indicates also the average frequency of the solids concentration fluctuations (van der Stappen et al., 1993a). Thus, the average cycle time represents the time scales in the fluidization system. This quantity is comparable to the average dominant frequencies derived from the spectral analysis (van der Stapen, 1993a, b). The distribution and range of cycle times or the times between crossings gives a measure of complexity and predictability of the multiphase flow (Daw and Halow, 1993). Literature shows that this concept is not new in fluidization engineering, but its power has not been fully exploited. Schouten and van der Bleek (1998) proposed the use of the average cycle time in controlling the fluidization quality. Daw and Halow (1993) used unidirectional crossing-time variability to calculate the Kolmogorov entropy using probability considerations. 
By recording the solids concentration fluctuations, it is possible to study the cluster dynamics in gas-solids CFB (Manyele et al., 2002; Tuzla et al., 1998; Soong et al., 1993). Clusters were identified in the time series based on the following three necessary conditions proposed by Soong et al. (1993): (a) The value of $\mathcal{E}_{s}$ must be significantly above the average solids concentration at the given local position for the given operating condition. A value of $1.5 \sigma$ was used to denote high concentration above the mean, where $\sigma$ is the standard deviation of the signal. (b) This perturbation due to cluster must be greater than the random fluctuations in the background of $\varepsilon_{s}(t)$ and exist for longer time than three times the sampling rate. (c) The perturbation must be sensed for sampling volume with characteristic length scale greater than one or two orders of particle diameter. Using sensitivity analysis, Manyele et al. (2002) further elucidated these criteria.

Important parameters from cluster identification studies include the cluster time fraction, $F_{c}$, and the average cluster existence time, $T_{c}$ (Manyele, et al., 2002). Other parameters include cluster frequency and cluster solids concentration. Based on these parameters it is possible to compare the dynamics between the two reactors. The cluster time fraction was determined by summing up the time intervals for which each cluster existed. The ratio of this sum to the total sampling time, $T_{s}$, expressed in percent, gives the time fraction, $F_{c}$, as per equation

$$
F_{c}=\frac{1}{T_{s}} \sum_{j=1}^{N_{c}}\left(T_{f}-T_{i}\right) \times 100 \%
$$

Where $T_{i}$ and $T_{f}$ are the initial and final times during which the cluster existed, that is, when $\varepsilon_{s}(t)$ existed above $\left(\varepsilon_{s}+1.5 \sigma\right)$ for more than three sampling time intervals.

The mean cluster existence time, $T_{c}$, is the average time interval between initial and final detection of different clusters in the signal. A time series of existence or residence times at a local position was created from the solids concentration signal. This time series was then processed for average existence time. Given the number of observed clusters at a local position, $N_{o c}$, the local mean existence time was determined according to the equation

$$
\tau_{c}=\frac{1}{N_{o c}} \sum_{j=1}^{N_{c}}\left(T_{f}-T_{i}\right)
$$

Other measures of complexity of multiphase reactor dynamics derived from deterministic chaos analysis include the correlation dimension (Manyele et al., 2003; van der Stappen et al. 1993a, 1993b; Daw and Hallow, 1993), and Kolmogorov entropy (Schouten et al., 1994b; Grassberger and Procaccia, 1983a; Grassberger and Procaccia, 1983b). The first step was to reconstruct attractors from the measured time series of solids concentration fluctuations.

The correlation dimension is a measure of complexity of the reconstructed attractor (which maps the gas-solids flow signals as trajectories in a multidimensional phase-space). The minimum embedding dimension where the attractor becomes fully developed is a measure of the number of dynamical degrees of freedom that govern the dynamics of gas-solids flow in the reactor. The correlation dimension was computed from the correlation integrals using a method of Grassberger and Proccaccia (1983a), following optimization of the time delay and number of embedding dimensions. Schouten et al. (1994a) and many others gave more details on the computation of correlation integrals and correlation dimension. Moreover, the minimal sufficient embedding dimension was determined by using the false nearest-neighbors algorithm (Hegger et al., 1999). The correlation integral, $C_{r}$, was computed from the equation derived by Grassberger and Proccaccia (1983a), as

$$
C_{r}=\frac{1}{M^{2}}\left(\begin{array}{l}
\text { number of pairs } \left.(i, j),\left|X_{i}-X_{j}\right|\right) \\
<r
\end{array}\right)
$$


where $X_{i}=$ multi-dimensional reconstructed (embedded) vector; $M=$ Number of points along the reconstructed trajectory; and $r$ is the scaling distance. The correlation dimension, $D$, is then computed as the slope of the linear portion of the $\log$-log plot of $C_{r}$ versus $r$, simplified as per equation

$$
C_{r}=r^{D}
$$

Thus, a higher correlation dimension, which signifies a more complex attractor, represents a more complex flow dynamics at the source of the signals, and vice versa (Fuller et al., 1996).

The Kolmogorov entropy, on the other hand, characterizes the sensitivity of the gas-solids flow to small disturbances and its rate of information loss; it is also a measure of the predictability of the changes in the solids concentration (Grassberger and Procaccia, 1983b). The most acceptable algorithm used to compute the Kolmogorov entropy from the time series is the maximum likelihood method developed by Schouten et al. (1994b).Using this algorithm, the Kolmogorov entropy, $K_{\mathrm{ML}}$ is defined as

$$
K_{M L}=-\frac{1}{\tau} \ln \left(1-\frac{1}{\bar{b}}\right)
$$

where $\bar{b}=$ average number of steps before the scaling distance, $r$, exceeds the average absolute deviation (AAD), and $\tau=\Delta \mathrm{t}$, the sampling time interval, (sec). The AAD is simply the average of the absolute values of the departure of the instantaneous values of the solids concentration from the mean value (Schouten and van den Bleek, 1998; Marzocchella et al., 1997; van der Stappen et al., 1993b), expressed AAD mathematically as

$$
A A D=\frac{1}{N} \sum_{i=1}^{N}\left|\varepsilon\left(t_{i}\right)-\overline{\varepsilon_{s}}\right|
$$

A positive value of Kolmogorov entropy is a necessary condition for a system to be chaotic. Higher entropy signifies higher rate of information loss (or lower predictability) and also shows stronger dependency on small disturbances. These two parameters have gained acceptance in characterizing the dynamical features of the chaotic dynamics of the CFB risers and downers.

Using these parameters the change in the dynamics with operating conditions (gas velocity and solids flux) was studied at different axial elevations. The second step was to relate the chaos parameters with the characteristics of multiphase flow in the two reactors.

\section{RESULTS AND DISCUSSION}

The dynamics of the CFB are embedded in the time series of the operating variables (Daw et al., 1990, and Huilin et al., 1995). Examining the temporal variations of solids concentration, $\varepsilon_{s}(t)$, reveals more details than that of pressure fluctuations due to the difference in measurement volumes between the two measurement methods. Moreover, the parameters derived from signal analysis contain more details compared to time-averaged data. Figure 2 shows the sample signals recorded using a fiber optic probe positioned at the center of the column $(r / R=0)$ for both downer and riser. The selected axial elevations depict entrance and fully developed sections with similar values of $z / Z$. The top or entrance section of the downer gives a signal with small variations in amplitude, despite of comparable mean values. However, the signal from the fully developed section of the downer shows highest amplitude. In the riser, the fluctuations of solids concentration are similar for both elevations, probably because of the strong driving force (high gas velocity), which is the same throughout the riser height along the center.

The difference in the strength of fluctuations in the entrance sections is mainly due to the fact that solids enters the downer via tubes emanating from a bed at minimum fluidization, implying that the jets travels in the entrance with same 
properties before they start mixing intensively downwards. This leads to uniform solids concentration signals in this section. In the riser, however, the gas-solids interactions at the entrance are very strong leading to strong fluctuations. corresponding to $0.4<r / R<0.85$ and $r / R>0.85$. In region I $(r / R<0.4)$, the radial profiles of $\bar{\varepsilon}_{\mathrm{s}}$ are relatively flat for both riser and downer, as expected for central regions, based on the principles governing flow in circular conduits. For region II, $0.4<r / R<0.85$, the downer is

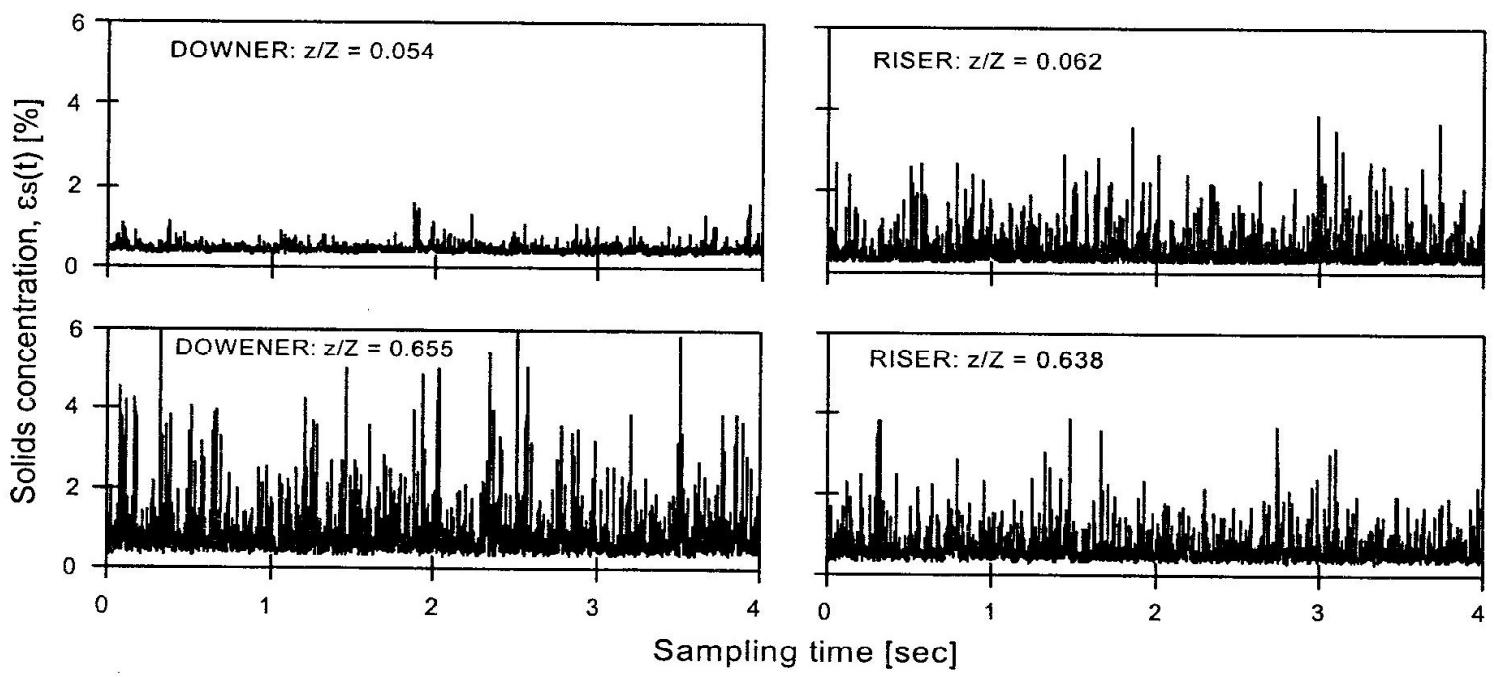

Figure 2: Sample solids concentration signals from similar axial elevations in the downer and riser at similar operating conditions $\left(U_{g}=8 \mathrm{~m} / \mathrm{s}, G_{s}=100 \mathrm{~kg} / \mathrm{m}^{2} \mathrm{~s}\right)$, at $r / R=0$.

In the fully developed sections with similar local solids concentration (approximately $0.66 \%$ ), the downer shows stronger fluctuations than riser, attributed to the fact that downer contents continues to accelerate downwards under the influence of gravity and gas flow, leading to stronger fluctuations than in the riser, where the solids attains a uniform velocity, and hence a steady flow.

The signals shown in Figure 2 are few samples selected out of 11 radial locations, denoted as $r / R$ (where $r$ is the actual radial position and $R$ is the diameter of the column). By computing the mean values of the signals (called time-averaged data) from all 11 radial positions, the radial variations of the solids concentration in the two reactors were studied, as shown in Figure 3.

The radial profiles of the time-averaged solids concentration are shown for the two elevations also at similar operating conditions. From the plot, differences in the time-averaged solids concentration are noticeable in the radial regions denser than the riser for both elevations. In the wall region, however, the profiles of solids concentration from both riser and downer are completely different. Even the profiles from the same reactor are different for different sections. Moreover, both profiles from the downer peaks in region II, while those from the riser entrance peaks in region III. In short, the variation of solids concentration with radial position is much more significant for riser than for downer in the fully developed region, while more variation occur in downer than in riser for the entrance regions. The downer entrance, however, shows stronger variations in the radial flow structure than the riser with higher solids concentration for regions II and III. That is, the flow structure of the riser and downer is non-uniform for all elevations, as reported also by $\mathrm{Zhu}$ and Manyele (2001).

Based on the time histories, $\varepsilon_{s}(t)$, different time series analysis techniques were used in comparing the dynamics of the downer and riser. The probability density function (PDF) shows 
the probability of finding different values of solids concentration in the signal. Higher peak in the PDF signifies highly uniform gas-solids flow with weak fluctuations from the mean, while flat PDF signifies highly fluctuating gas-solids flow. Because the shape of the PDF reveals differences in the dynamics between different signal sources, this method was used to compare the dynamics of the two reactors. Figure 4 compares the PDFs from similar locations in the downer and riser. Note that the solids concentration on the horizontal axis is very low, signifying a dilute core region in both cases (see also Figure 3).

The PDF depicted in Figure 4 can be
The highest peak for a signal from the downer entrance reveals a weakly fluctuating solids concentration as shown also in Figure 2. This shows that most values are closer to the mean value of $0.44 \%$. The signal from the entrance of the riser, however, shows a shortest peak in the PDF indicating that the solids concentration fluctuates strongly with time, mainly due to the following factors: the jetting effect of the gas from the riser distributor holes; the instability of flow at the bottom due to acceleration of solids; and the refluxing tendency of solids in this section. The fully developed regions of both riser and downer show similar behavior (as shown

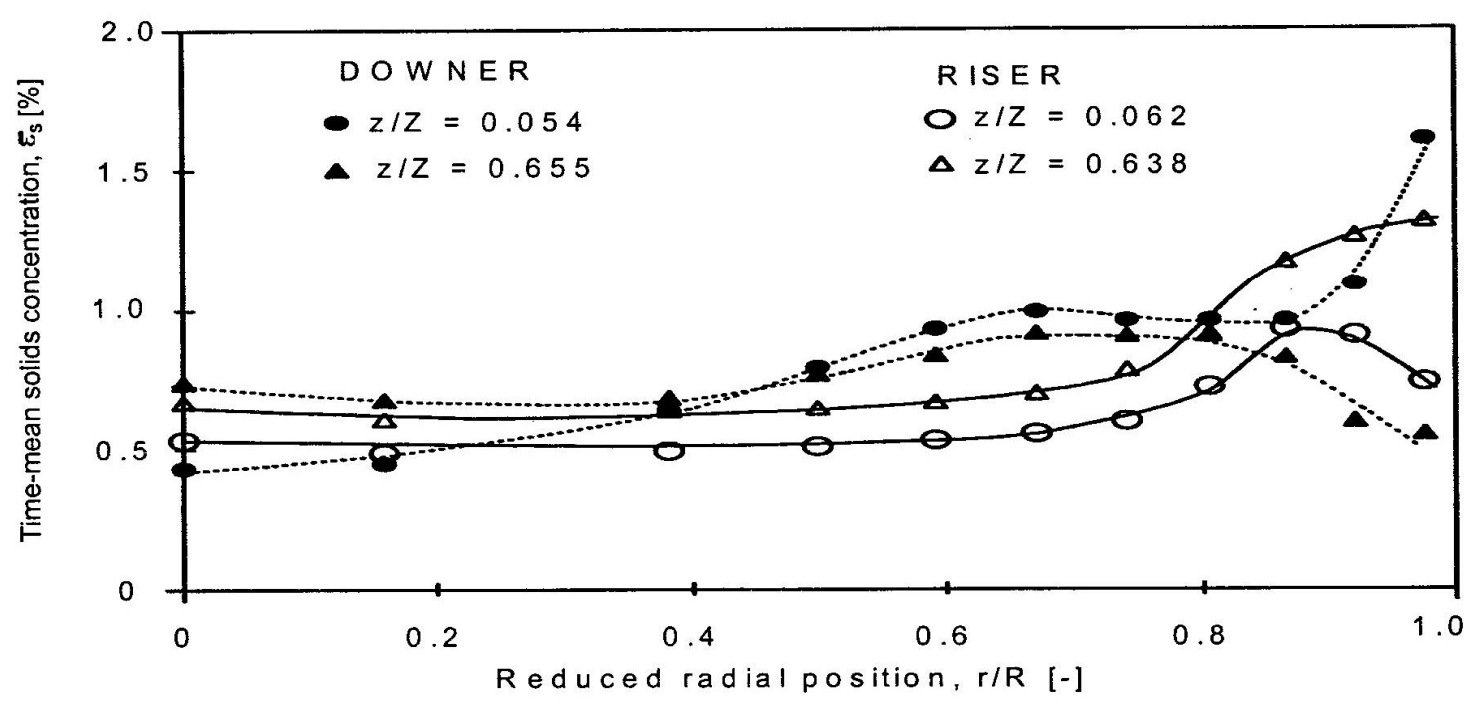

Figure 3: Radial profiles of average solids concentration in the entrance and fully developed regions of downer and riser reactors at similar operating conditions $\left(U_{g}=8 \mathrm{~m} / \mathrm{s}, G_{s}=100 \mathrm{~kg} / \mathrm{m}^{2} \mathrm{~s}\right)$.

characterized as gamma type (Johnson and Johnson, 2001). The most important feature of these PDFs is the long tails towards higher values of $\varepsilon_{s}$. This is due to presence of solids aggregates or clusters in the CFB system utilizing fine powders (Manyele et al., 2002). While the $\varepsilon_{s}$ is low, the passage of clusters of high solids concentration at a given location gives higher values of $\varepsilon_{s}$ periodically and hence long tails in the PDF. Hence, the long tails at solids concentration higher than $0.8 \%$ corresponds to the cluster phase. Despite the low frequency of $\varepsilon_{s}$ values in this range, the effect of this phase on the reactor dynamics and hence its performance, is substantial. also in Figure 2), where similar peaks can be seen with equally long tails, indicating a similar flow pattern. The probability for $\varepsilon_{s}>0.8 \%$ is generally small, but not zero. This non-zero probability indicates presence of clusters, as observed for both riser and downer. The high probability values at higher solids concentration for the downer at $z / Z=0.655$ indicates higher tendency of clusters formation in the downer compared to riser, mainly due to the accelerating tendency of solids in the fully developed region $f$ the downer and hence cluster formation according to principle of energy minimization. Moreover, clusters in the downer can take large 


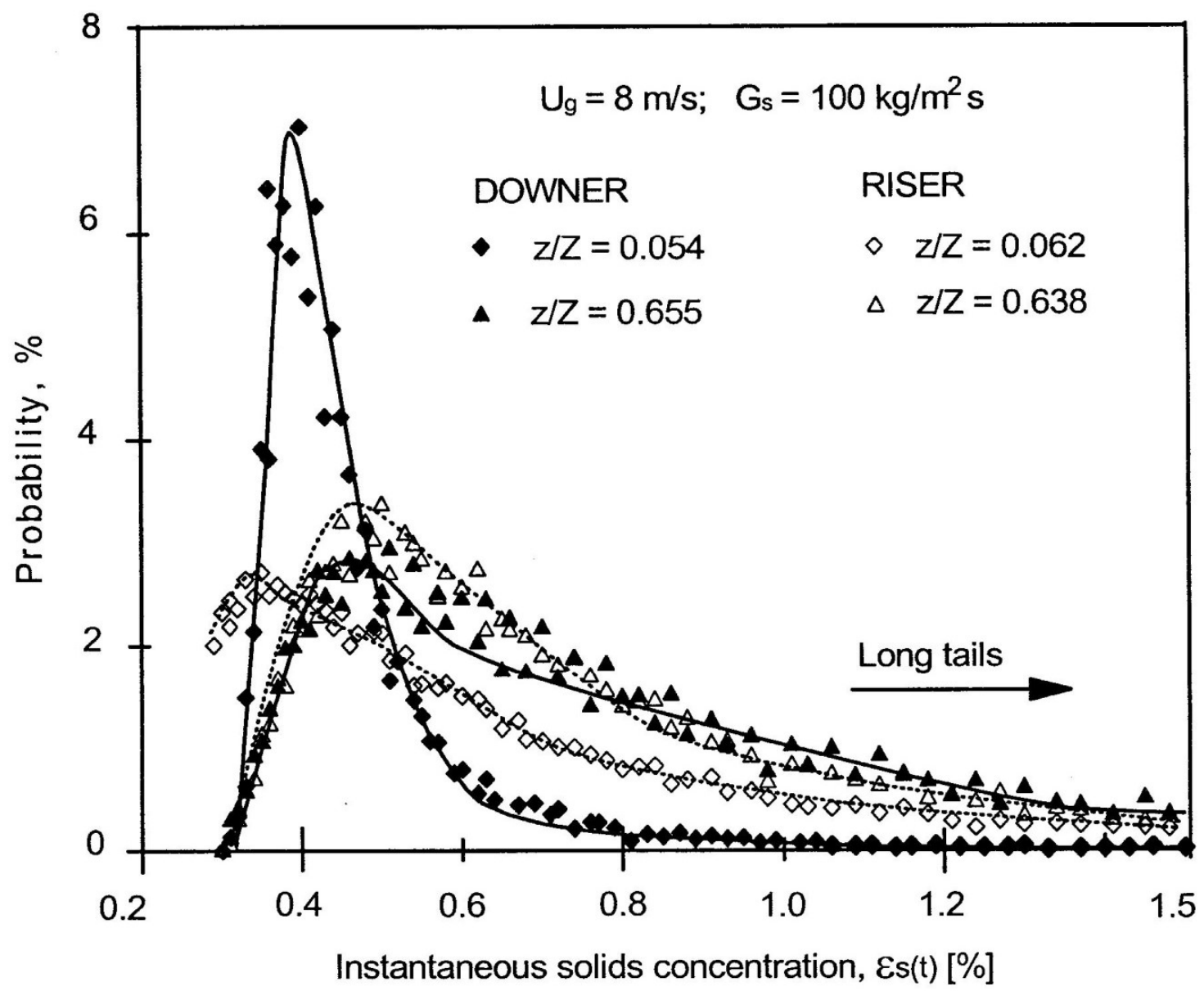

Figure 4: Probability distribution plots of solids concentration signals for downer and riser (center positions) at selected axial locations, $z / Z$, and $r / R=0$, at $U_{g}=8.0 \mathrm{~m} / \mathrm{s}$ and $\mathrm{G}_{\mathrm{s}}=100 \mathrm{~kg} / \mathrm{m}^{2} \mathrm{~s}$.

sizes due to the free-fall nature of flow for the solids and gas.

The standard deviation is a commonly used measure of variation in the signals. In both riser and downer, the standard deviation of solids concentration fluctuations depends on the suspension density, gas turbulence and particleparticle collisions. However, the intensity of solids concentration fluctuations does not increase indefinitely with increasing solids concentration (Issangya et al., 2000). The fluctuations decrease again as the mean solids concentration increases. The plot of the standard deviation versus time-averaged solids concentration gives a quadratic relationship, with a maximum for solids concentration beyond $10 \%$, under conditions of high density CFB
(Issangya et al., 2000). Such plots can be used to compare different multiphase reactors from the way the standard deviation varies with the timeaveraged solids concentration. Figure 5 is a plot of such a relationship for both riser and downer using data from different radial position and different operating conditions (increasing gas velocity at constant solids flux for all radial positions).

According to Figure 5, the solids concentration have a shorter span for downer $(0.5-1.05 \%)$ but wider range for riser $(0.5-10 \%)$ in the fully developed sections. For the same solids concentration range which exists in both riser and downer, the standard deviation is the same, such that the data points coincide on the same curve. This was observed for solids 
concentration in the range of $0.5-1.05 \%$. Moreover, higher standard deviation valucs cxist in the riser, where $\varepsilon_{s}$ values are higher under similar operating conditions of solids flux and gas velocities. This corresponds to the wall region of the riser. All the data points fall on the same curve for both riser and downer as shown in the small insert where the data points are combined. The observation that the curve indicates presence of a maximum in standard deviation for $\varepsilon_{s}>10 \%$, is in accordance to the observation reported by Issagya et al. (2000).

It is evident from Figure 5 that the fluctuations are stronger in the riser than downer. Also, the span of solids concentration is wider in the riser than in the downer due to wall effect in the riser. Tuzla et al. (1998) reported also that $\bar{\varepsilon}_{\mathrm{s}}$ was higher in riser than in downer at all radial positions, for $Z=4 \mathrm{~m}$, which agrees well with results shown in Figure 5.

In both downer and riser, the standard deviation of the signal depends on the suspension density, gas turbulence and particle-particle collisions. The major difference between upflow and downflow CFBs is the range of standard deviation. However, it is interesting to observe that the data follows the same curve for both upflow and downflow CFB reactors. The effect of operating conditions on the dynamics of the two kinds or reactors can be revealed from Figure 5. In the riser high gas velocity lowers the intensity of fluctuations (low standard deviation at $U_{g}=8.0 \mathrm{~m} / \mathrm{s}$ ), while in the downer the intensity of solids concentration fluctuations increases with gas velocity.

The signals of solids concentration were also characterized using the average cycle time and the number of cycles. These two parameters have wide applications in the analysis of the dynamics of multi-phase reactors, use of which necessitates that the signal be stationary, as shown in Figure 2. Figure 6 shows the radial distribution of the average cycle time, $T_{a c}$ and number of cycles, $N_{c}$, for the entrance and fully developed regions.

In the core region, the values of $T_{a c}$ are slightly low in riser than in the downer, showing that at similar locations the changes in solids concentration takes place faster in riser than in the downer. In the wall region, the difference in $T_{a c}$ values is magnified, whereby the values increase for downer and decrease faster for riser, indicating stronger interaction of the gas and solid phases near the wall of the riser. It is evident that the energy content of the flow is higher in the riser than in the downer. The difference in average cycle time is more pronounced in the wall regions of riser and downer, mainly due to downflow of solids near the wall of the riser. This observation is in accordance with the literature reports that the wall region of the riser in the fully developed region experiences stronger fluctuations due to downflow of solids (Zhu and Manyele, 2001). 


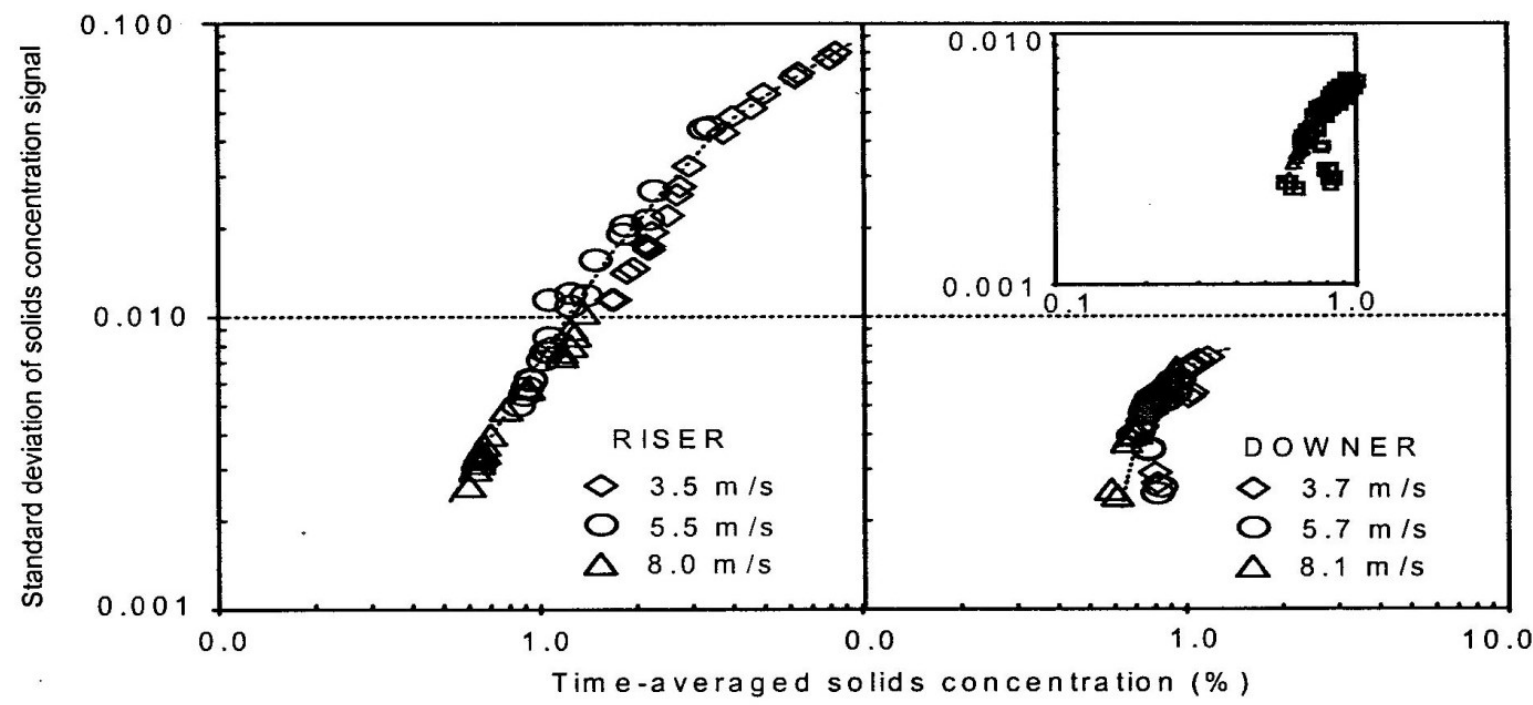

Figure 5: Effect of gas velocity on the intensity of solids concentration fluctuations (expressed as standard deviation versus average values) in the fully developed regions of downer and riser at $G_{s}=100 \mathrm{~kg} / \mathrm{m}^{2} \mathrm{~s}$, and different gas velocities

There is little difference observed in the number of cycles between riser and downer, except in the wall region, where the downer shows fewer crossings on the mean value. This signifies that changes occur slowly in the downer. Because of fewer cycles in the wall region of the riser, shorter cycle times, and these results indicates a phenomenon that needs further research with a wide range of data.

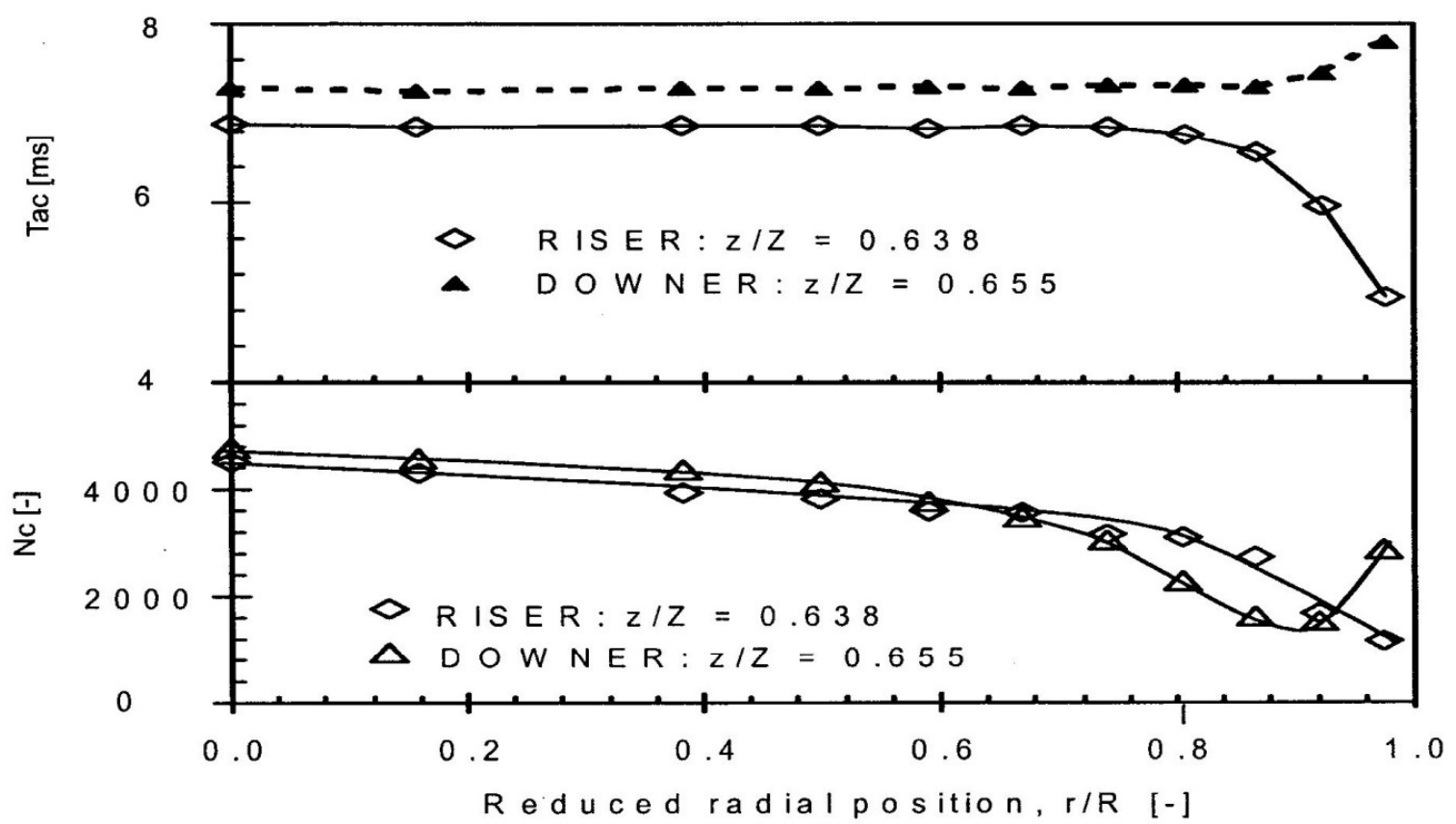

Figure 6: Radial variation of average cycle time, $T_{a c}$, and number of cycles, $N_{c}$, (within $16 \mathrm{sec}$ ) in the solids concentration signal from downer and riser in the fully developed regions at $U_{\mathrm{g}}=8.0$ $\mathrm{m} / \mathrm{s}$ and $G_{\mathrm{s}}=100 \mathrm{~kg} / \mathrm{m}^{2} \mathrm{~s}$. 
Cluster properties defined above were used to compare the dynamics of of the two reactors. Figure 7 shows the radial profiles of $F_{c}$ and $\tau_{c}$, for both riser and downer in the fully developed regions.

The values of $F_{c}$ increase near the wall for both reactors, indicating a higher tendency towards cluster formation near the wall. However, the analysis was used for further comparison of the complexity and predictability of the flow between the two reactors. The radial profiles of correlation dimension at $U_{g}=8.0 \mathrm{~m} / \mathrm{s}$ and $G_{s}=$ $100 \mathrm{~kg} / \mathrm{m}^{2} \mathrm{~s}$ in the fully developed-flow and entrance sections of the riser and downer are shown in Figure 8.

The range of the values of correlation dimension

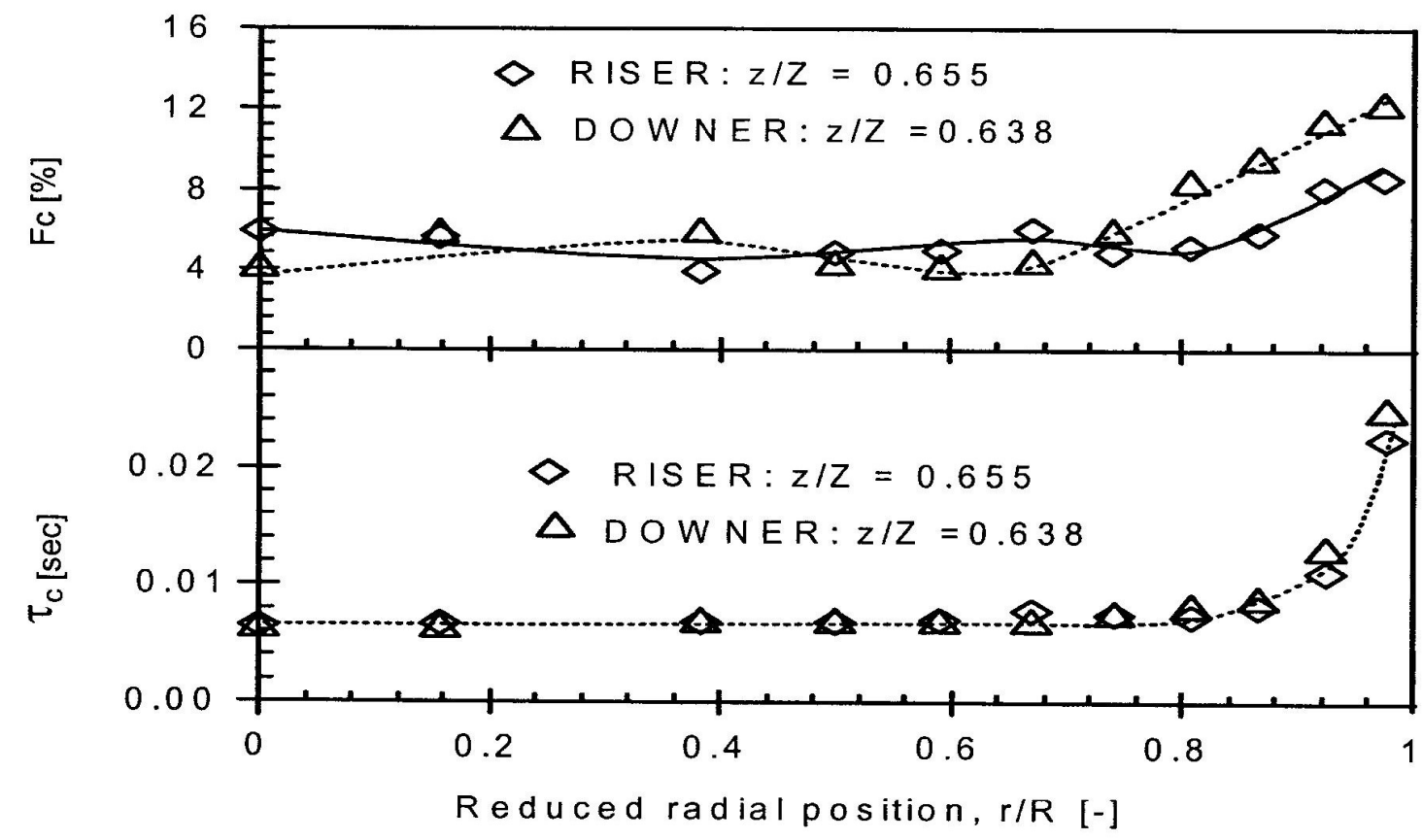

Figure 7: Radial variation of fraction of time occupied by clusters $F_{c}$, and mean residence time of clusters, $\tau_{c}$, for downer and riser in the fully developed regions at $U_{g}=8.0 \mathrm{~m} / \mathrm{s}$ and $G_{s}=$ $100 \mathrm{~kg} / \mathrm{m}^{2} \mathrm{~s}$.

values of $F_{c}$ are lower in the wall region of riser than in the downer, due to breakdown of clusters in the riser caused by gas flow. In both reactors, clusters were observed at all radial positions in less than about $15 \%$ of the time. The values and radial profiles of $\tau_{c}$ were found to be similar in the two reactors. Shortest duration of the cluster existence was observed at the center of the columns, as reported also by Tuzla et al. (1998). Thus, in terms of cluster existence times, the two reactors show similar results. The major difference is the cluster frequency, which manifests in the values of $F_{c}$.

As a result of clusters, gas-solids interaction and gas-solids-wall interactions, the time variation in flow becomes complex and chaotic. Thus, chaos in both riser and downer is comparable. The differences arise in the profiles especially in the region from $r / R>0.6$. In both cases, there is a characteristic decrease followed by a sharp increase in the complexity of multiphase flow (expressed by values of $D$ ) in both reactors. The decrease in $D$ near the wall can be attributed to the high solids concentration in this region, which compensates the solids concentration fluctuations by damping effect, while the sharp increase in $D$ at the wall can be attributed to the solids-wall interactions. This observation conforms to the literature report by Chen et al. (1995) that $D$ increases near the wall of the downer, despite a difference in operating conditions. In the entrance section, however, $D$ 


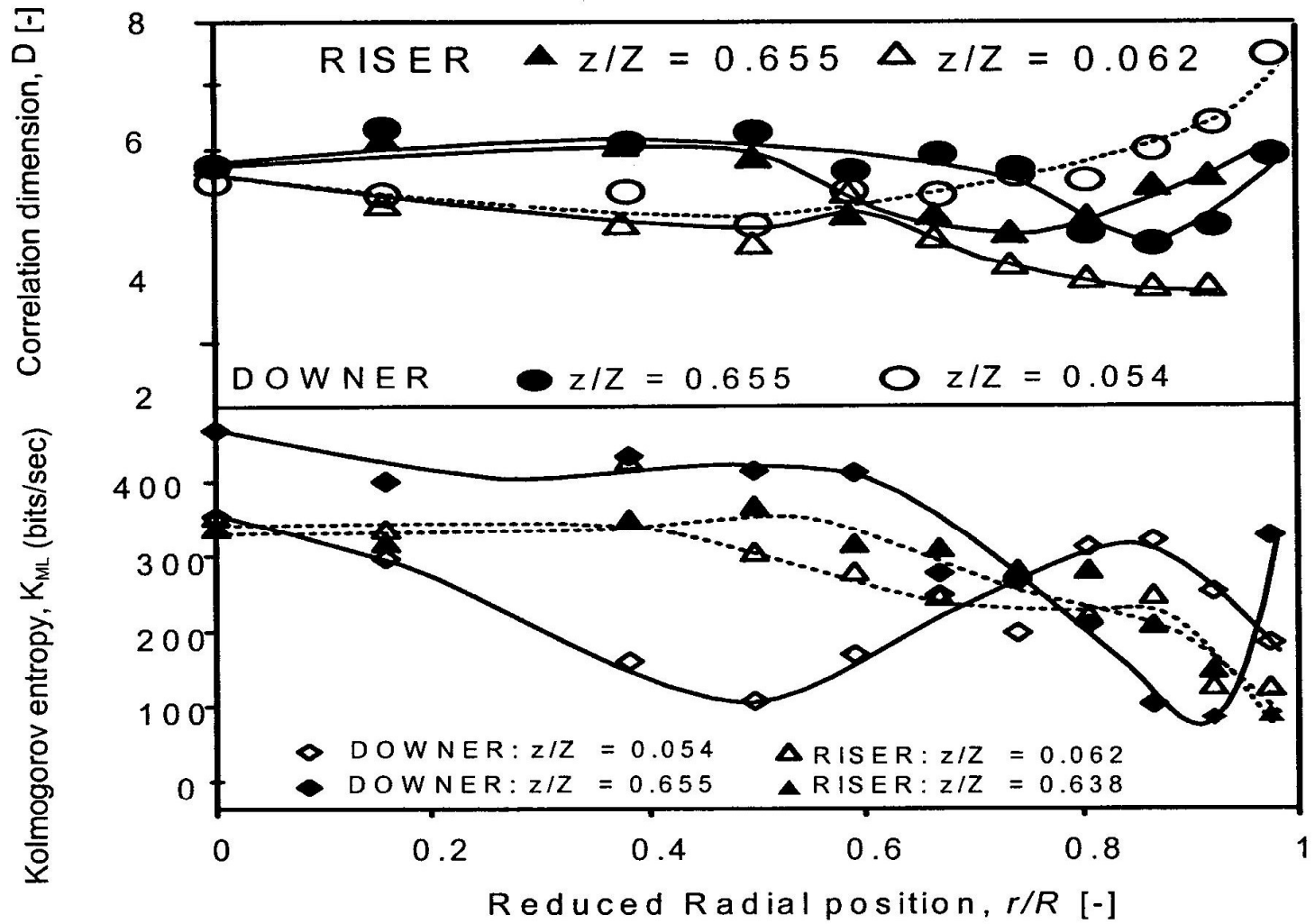

Figure 8: Radial profiles of correlation dimension, $D$, for solids concentration time series from fully developed regions of downer and riser at similar operating conditions $\left(U_{g}=8.0 \mathrm{~m} / \mathrm{s}\right.$ and $G_{s}$ $\left.=100 \mathrm{~kg} / \mathrm{m}^{2} \mathrm{~s}\right)$.

decreases towards the wall in the riser while it increases for the case of downer. This can be attributed to the difference in the gas-solids feeding methods between the two reactors. At the wall of the downer entrance, the solids are free to move randomly (leading to higher values of $D$ ) different from the riser, where a dense bed exists near the wall at the entrance, causing lower values of $D$.

Figure 8 shows also the radial profiles of Kolmogorov entropy, $K_{\mathrm{ML}}$, under similar operating conditions. It should be noted that all values of $K_{\mathrm{ML}}$ are positive, which indicates chaotic flow. The radial profiles of $K_{\mathrm{ML}}$ show remarkable differences. Higher values of $K_{\mathrm{ML}}$ in the core region for both reactors are due to the prevailing dilute conditions which lead to free solids motion. In the entrance section, both reactors show peaks in the radial profiles around $r / R=0.8$. In the fully developed flow sections, the $K_{\mathrm{ML}}$ decreases towards the walls of both riser and downer, despite a sharp rise at the wall of the downer. In general terms, the solids motion in the riser and downer is more complex and chaotic in the core than in the wall, and also it is less predictable in the core than in the wall region.

\section{CONCLUSION}

The microscopic flow dynamics in downer and riser have been analyzed extensively in this study. While the major cause for the differences include gravity, the solids and gas feeding mechanism, entrance effects, etc., in particular, the flow development plays a big role affecting the flow dynamics of the two reactors in different ways. Because all the effects caused by differences in operating conditions, column diameters and nature of powder were eliminated, 
this study gives a more dependable analysis, than literature reports.

In particular, the following conclusions can be made:

The major differences in the dynamics of the two reactors occur in the wall regions, and in the entrance sections.

The dynamics in the fully developed regions of both riser and downer are similar in the core regions.

The shorter cycle times for the signals observed in the riser, supports the fact that the fluctuations of flow parameters in riser are more intense than in the downer.

The range of cluster mean residence time in both riser and downer is comparable except at the wall, where downer has longest duration of cluster residence time.

For the given sampling duration, the cluster-time fraction was found to be the same in the core regions of downer and riser but was higher for downer in the wall region for the fully developed regions.

Chaos analysis has revealed also that the particle movement is more chaotic in the core of the two reactors than in the wall regions, and that the range of values of the correlation dimension and Kolmogorov entropy are comparable.

\section{NOMENCLATURE}

$\bar{b} \quad$ Average number of steps before the distance exceeds $\mathrm{AAD}$ on the attractor $[-]$

$C_{\mathrm{r}} \quad$ Correlation integral [-]

$D \quad$ Correlation dimension [-]

$F_{\mathrm{c}} \quad$ Cluster time fraction [-]

$G_{\mathrm{s}} \quad$ Solids circulation rate $\left[\mathrm{kg} / \mathrm{m}^{2} \mathrm{~s}\right]$

$M \quad$ Number of points in the reconstructed vector [-]

$N_{\mathrm{c}} \quad$ Number of cycles [-]

$N_{o c} \quad$ Number of clusters [-]

$r \quad$ Scaling distance in phase space [-] $r / R \quad$ Reduced radial position [-]

$T_{c} \quad$ Mean residence time of clusters [sec]

$T_{f} \quad$ Final time of cluster existence [sec]

$T_{i} \quad$ Initial time of cluster existence [sec]

$T_{a c} \quad$ Average cycle time [sec]

$U_{g} \quad$ Superficial gas velocity $[\mathrm{m} / \mathrm{s}]$

$X \quad$ Multi-dimensional reconstructed vector [-]

$Z \quad$ Axial elevation starting from entrance $[\mathrm{m}]$

$z / Z \quad$ Reduced axial position [-]

Greek letters

$\bar{\varepsilon}_{\mathrm{s}} \quad$ Time-average solids concentration [-]

$\varepsilon_{\mathrm{s}}(\mathrm{t}) \quad$ Solids concentration time series [-]

$\Delta T_{\mathrm{c}} \quad$ Crossing time interval [sec]

$\tau \quad$ Sampling time interval, $\Delta t[\mathrm{sec}]$

$\tau_{c} \quad$ Cluster existence time [sec]

\section{REFERENCES}

1. Bai, D., J.-X. Zhu, Y. Jin, and Z. Yu, "Internal Recirculation Flow Structure in Vertical Upflow Gas-Solid Suspensions. Part II. Flow Structure Predictions." Powder Technol., 85, 179-188, (1995).

2. Bolton, L.W. and J.F. Davidson, "Recirculation of Particles in Fast Fluidized Beds". In Circulating Fluidized Bed Technology II, P. Basu and J.F. Large (Eds.), Pergamon Press, Oxford, 1988, pp. 139-152.

3. Cheng, Y., F. Wei, Q. Lin, and Y. Jin, "A Comparison of Local Chaotic Behaviors in a Riser and a Downer", in "Fluidization IX", Fan, L.-S. and Knowlton, T.M. (Eds.), Engineering Foundation, NY, (1998), pp. 613-620.

4. Daw C.S. and Halow J.S., (1993). "Evaluation and Control of Fluidization Quality through Chaotic Time Series Analysis of Pressure-drop Measurements", AIChE Symposium Series 89(296), pp. 103122. 
5. Daw C.S., W.F. Lawkins, D.J. Downing and N.E.Clapp Jr., (1990). "Chaotic Characteristics of a Complex Gas-Solids Flow", Physical Review A, 41(2), pp. 11791181.

6. Fuller, T.A., T.J. Flynn, and C.S. Daw, "Analysis of Dynamic Boiler Measurements: A Practical Approach", Chem. Eng. J., 64, 179-189 (1996).

7. Grassberger, P. and I. Procaccia, "Estimation of the Kolmogorov Entropy from a Strange Signal", Physical Review, A28, 2591-2594 (1983b).

8. Grassberger, P. and I. Procaccia, "Measurement of Strangeness of the Strange Attractors". Physica, 9D, 189-208 (1983a).

9. Hegger, R. H. Kantz and T. Screiber, "Practical Implementation of Nonlinear Time Series Methods: The TISEAN Package, Chaos, 9, 413 (1999).

10. Huilin L., D. Gidaspow, J.X. Bouillard, "Dimension Measurements of Hydrodynamic Attractors in Circulating Fluidized Beds", AIChE Symp. Ser., 91, 103-111 (1995).

11. Issangya, A.S., J.R. Grace, D. Bai, and J.-X. $\mathrm{Zhu}$, "Further Measurements of Flow Dynamics in a High-Flux CFB Riser", Powder Technol., 111, 104-113 (2000).

12. Johnson, F.A., A. Svensson, and B. Leckner, (1992), "Fluidization Regimes in CFB Boilers". In Fluidization VII, O.E. Potter and D.J. Nicklin, (Eds.), Engincering Foundation, NY, pp. 471-478.

13. Johnson, H. and F. Johnson, (2001), "Measurement of Local Solids Volume Fraction in Fluidized Bed Boilers", Powder Technol., 115, 13-26.

14. Manyele, S.V., J.H. Pärssinen and J.-X. Zhu, "Characterizing Particle Aggregates in a High-Density and High-Flux CFB Riser", Chem. Eng. J., 88 (2002) 151-161.

15. Manyele Samwel, V., Jesse Zhu, and Hui Zhang, "Analysis of the Microscopic Flow Structure of a CFB Downer Reactor Using Solids Concentration Signals", International
Journal of Chemical Reactor Engineering, Volume 1, Article A55, Produced by The Berkeley Electronic Press, 2003, pp. 1-17, http://www.bepress.com/ijcre/voll/A55

16. Marzochella, A, R.C. Zijerveld and J.C. Schouten, "Chaotic Behavior of Gas-Solids Flow in the Riser of a Laboratory-Scale Circulating Fluidized Bed", AIChE J., 43, 1458-1468 (1997).

17. Schouten , J.C., F. Takens and C.M. van den Bleek, "Estimation of the Dimension of a Noisy Attractor", Phys. Rev. E, 50(3), 18511861 (1994a).

18. Schouten, J.C., F. Takens and C.M. van den Bleek, "Maximum Likelihood Estimation of the Entropy of an Attractor", Phys. Rev. E, 49(1), 126-129 (1994b).

19. Schouten, J.C. and C.M. van den Bleek, "Monitoring the Quality of Fluidization using short-term Predictability of Pressure Fluctuations", AIChE J., 44(1), 48-60, (1998)

20. Soong, C. H., K. Tuzla, and J.C. Chen, "Identification of Particle Clusters in Circulating Fluidized Bed", in "Circulating Fluidized Bed Technology IV", A. A. Avidan, (Ed.), AIChE, New York, (1994), pp. 615-620.

21. Svensson, A., F.A. Johnson, and B. Leckner, (1996), "Fluidization Regimes in NonSlugging Fluidized Bed: The Influence of Pressure Drop across the Riser Air Distributor", Powder Techn., 86, 299-312.

22. Tuzla, K., A.K. Sharma, J.C. Chen, T. Schiewe, K.E. Wirth and O. Molerus, "Transient Dynamics of Solids Concentration in Downer Fluidized Bed", Powder Technol., 100, 166-172 (1998).

23. Van der Stappen M.L.M., J.C. Schouten, and C.M. van den Bleek, "Application of Deterministic Chaos Theory in Understanding the Fluid Dynamic Behavior of Gas-Solids Fluidization", AIChE Symp. Ser., 89 (296), 91-102, (1993a).

24. van der Stappen, M.L.M., J.C. Schouten and C.M. van den Bleek, "Application of 
Deterministic Chaos Analysis to Pressure Fluctuation Measurements in $0.96 \mathrm{~m}^{2} \mathrm{CFB}$ Riser", Preprint of the $4^{\text {th }}$ Int. Conf. on $C F B s, 1993 b$, Somerset, Pennsylvania, pp. 55-60.

25. Zhang, H., P.M. Johnston, J.-X. Zhu, H.I. de Lasa, and M.A. Bergougnou, "A Novel Calibration Procedure for a Optical fiber Concentration Probe", Powder Technol., 100, 260-272 (1998).

26. Zhu, J.-X. and S.V. Manyele, (2001), "Radial Nonuniformity Index (RNI) in Fluidized
Beds and other Multiphase Flow Systems", Can. J. Chem. Eng., 79(2), 203-213.

27. Zhu, J.-X. and S.V. Manyele, (2001), "Radial Non-Uniformity Index in Fluidized Beds and other Multiphase Flow Systems", Can. J. of Chem. Eng., Vol. 79, 20-213.

28. Zhu, J.-X., Y. Jin, Z-Q. Yu, J.R. Grace, and A. Issangya, "Concurrent Downflow Circulating Fluidized Bed (Downer) Reactors - A State of the Art Review", Can. J. Chem. Eng., 73, 662-677, (1995). 